\title{
Mammographic, ultrasonographic and pathologic correlations of focal asymmetric breast densities among a sample of iraqi women
}

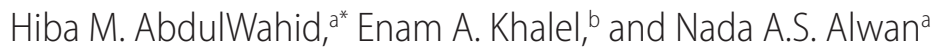 \\ aNational Cancer Research Center, University of Baghdad, Baghdad, Iraq. \\ bRadiology Department, Oncology Teaching Hospital, Baghdad, Iraq. \\ *Correspondence to Hiba M. Abdul Wahid (email: dr.hiba.mohammed85@gmail.com). \\ (Submitted: 25 December 2018-Revised version received: 20 January 2019 - Accepted: 25 April 2019 - Published online: 26 June 2019)
}

\begin{abstract}
Objective The aim of the study is to evaluate the mammographic focal asymmetric breast densities (FABD) in order to highlight which FABD might need further workup through detailed ultrasonographic characterization and comparison with the pathological results in indicated cases.

Methods A cross-sectional analytic study was performed in the Oncology Teaching Hospital, Medical City from March 2018 to November 2018. The study included 70 women who attended the Main Referral Center for Early Detection of Breast Tumors with FABD were detected by mammography. The focal asymmetry was analyzed and the other associated findings were assessed and registered. While breast ultrasound was performed for all patients, fine needle aspiration (FNA) was carried out for cases with suspicious findings on ultrasound and any FNA suspicious or malignant lesion was subsequently biopsied and the results were recorded.

Results Mammographic FABD was found in the right breast in 37 cases (52.9\%) and in the left in 33 cases (47.1\%). The upper outer quadrant was the most common location of FABD (43 cases). In 25 cases (34.2\%) with FABD, there was no abnormality found on ultrasound, but normal looking breast parenchymal tissue. Ultrasound had shown benign findings in 30 cases (44.2\%). On the other hand, suspicious and/ or malignant features were observed in 15 cases (22.4\%) which subsequently proved to be malignant by FNA and biopsy. Architectural distortion or grouped microcalcifications and a clinically palpable FABD were associated with malignant FABD; no benign FABD revealed to be associated with these findings.

Conclusion The finding of FABD is common on mammography and mostly represents a benign entity of normal breast parenchymal tissue. However, it may indicate an underlying hidden malignancy especially in the presence of superadded mammographic findings as ill-defined mass, architectural distortion or grouped microcalcification.

Keywords mammography, ultrasonography, asymmetric breast density, Iraq
\end{abstract}

\section{Introduction}

Breast cancer is the most common malignancy in women over the age of 40 and the most reported cause of cancer death in women worldwide. ${ }^{1,2}$ It has been shown that the early detection and screening followed by appropriate management reduces the associated morbidity and mortality rates of the disease. ${ }^{3}$ Previous surveys from Iraq have reported that breast cancer is the most frequent registered malignancy ${ }^{4}$ and that most of the cases are often detected among middle aged women in relatively advanced stages. ${ }^{5-7}$ Mammography is an important screening tool in the early detection of breast cancers. ${ }^{8}$ It can detect about $75 \%$ of breast cancers at least a year before they become symptomatic. ${ }^{9}$

Mammogram interpretation is a time-consuming and sometimes considered as a difficult task due to a wide variability of the detected breast abnormalities and overlapping dense fibroglandular tissues. ${ }^{10}$ Although there is a great difference in the parenchymal patterns and sizes of breasts, the internal structures are nearly symmetrical with similar densities and architectures at mammography. Asymmetric breast densities are repeatedly seen at screening or diagnostic mammography and are usually nonspecific and common finding in healthy women, however; these findings are sometimes due to a hidden malignancy. ${ }^{11,12}$ Thus, it has been recommended that when asymmetric finding is detected on mammogram, it should be assessed carefully and further evaluation is needed to decide whether it reflects a normal variant or something that is more significant. ${ }^{13}$ The American College of Radiology-Breast Imaging Reporting and Data System (ACR-BI-RADS) has developed a lexicon for asymmetric breast densities. ${ }^{14}$ An asymmetric finding represents an area of breast tissue that has a fibroglandular density that is more extensive in one breast in comparison with the corresponding region in the contralateral breast while a mass is a three-dimensional lesion with convex outward margins and is usually seen on two orthogonal views. ${ }^{15}$

Asymmetric findings on mammogram were classified into four types according to the fourth edition of BI-RADS including: (1) Asymmetry as an area of fibroglandular tissue seen on one mammographic view frequently caused by overlapping of normal parenchymal tissue of breast; (2) global asymmetry which is seen over at least one quarter of the breast and is usually a normal variant; (3) developing asymmetry is a new larger and more conspicuous than on a previous examination ${ }^{16,17}$; and (4) focal asymmetry, the core of this study, which is defined as asymmetry of tissue density with similar shape on two views but has no borders and should be distinguished from a mass. It may represent true abnormality rather than superimposition. ${ }^{14}$

The aim of the study is to evaluate the mammographic focal asymmetric breast densities (FABD) in order to highlight which FABD might need further workup through detailed ultrasonographic characterization and comparison with the pathological results in indicated cases.

\section{Patients and Methods}

A cross-sectional analytic study was performed in the Oncology Teaching Hospital, Medical City from March 2018 
to November 2018. The study included 70 females who attended the Main Referral Center for Early Detection of Breast Tumors either for screening or diagnostic mammogram examinations, and FABD (as defined by the ACR-BI-RADS lexicon) had been detected on their mammograms.

All mammograms were performed using the Analog mammogram machine (Siemens). Two mammographic views were taken for each breast; mediolateral oblique and craniocaudal views. The mammographic images were interpreted by an experienced specialist radiologist. The focal asymmetry was analyzed and compared with previous mammograms (when available) searching for any change. Other associated findings were assessed and registered, these include: architectural distortion, grouped microcalcification and any associated mass lesion. Breast ultrasound was performed for all patients by using GE machine (Voluson E6 with linear transducer of frequency of 7.5-12 MHz) and ultrasound findings were also recorded (any mass or underlying suspicious area or only prominent fibroglandular tissue) and classified according to the ultrasound BI-RADS lexicon.

Fine needle aspiration was done for any suspicious finding on ultrasound, any fine needle aspiration (FNA) suspicious or malignant lesion was subsequently biopsied and the corresponding cytological and histopathological results were recorded.

\section{Statistical Analysis}

The collected data were tabulated and analyzed using Microsoft Excel 2010. The categorical data were presented as frequency and percentage tables. $P$-value $<0.05$ were considered as statistically significant.

\section{Results:}

During the study period, FABD were found in the mammograms of 70 female patients. Breast ultrasound was performed for all those patients. FABD was found in the right breast in 37 cases $(52.9 \%)$ and in the left in 33 cases (47.1\%). The upper outer quadrant (UOQ) was the most common location of FABD, it was found in 43 cases (61.4\%) followed by the retroareolar region in 15 cases $(21.4 \%)$. Other locations are summarized in Table 1.

In 25 cases $(35.7 \%)$ with FABD on mammogram, there was no abnormality found on ultrasound, but normal looking breast parenchymal tissue. Ultrasound had shown benign findings in 30 cases ( $42.8 \%$ ). Suspicious and/or malignant features were the sonographic findings in 15 cases $(21.4 \%)$ which subsequently proved to be malignant by FNA and biopsy. This was demonstrated in Table 2.

Ductal dilatation was the ultrasound finding in 12 cases of FABD (17\%) and was located in the retroareolar region with significant relation between retroareolar FABD and ductal dilatation $(P<0.05)$. Ultrasound showed cystic lesions in nine cases $(12.8 \%)$, seven of them (10\%) appeared as simple breast cysts, one (1.4\%) was a complicated cyst, and the other one (1.4\%) was a complex cystic lesion with a solid component invading the cyst wall (i.e. suspicious for malignancy; BI-RADS IV lesion). FNA and biopsy subsequently revealed breast cancer in the last complex lesion described.

Solid masses were the ultrasound findings in nine cases (12.8\%), four of them (5.7\%) appeared well defined and

\begin{tabular}{|c|c|c|}
\hline Location in either breast & No. & Percentage (\%) \\
\hline UOQ & 43 & 61.4 \\
\hline Retroareolar & 15 & 21.4 \\
\hline UIQ & 5 & 7.14 \\
\hline Midaspect & 4 & 5.7 \\
\hline LIQ & 3 & 4.28 \\
\hline Total & 70 & 100 \\
\hline
\end{tabular}

FABD, focal asymmetric breast densities; UOQ, upper outer quadrant; UIQ, upper inner quadrant.

\begin{tabular}{lccc}
$\begin{array}{l}\text { Table 2. Final diagnostic outcome according to ultrasound and } \\
\text { pathologic results }\end{array}$ & No. & $\begin{array}{c}\text { Percentage } \\
\text { (\%) }\end{array}$ & Note \\
\hline Final diagnostic outcome & 25 & 35.7 & $\begin{array}{l}\text { Depend on } \\
\text { US BI-RADS } \\
\text { classification } \\
\text { Depend on }\end{array}$ \\
\hline $\begin{array}{l}\text { Normal appearing } \\
\text { breast tissue by } \\
\text { ultrasound }\end{array}$ & 30 & 42.8 & $\begin{array}{l}\text { US BI-RADS } \\
\text { Classification } \\
\text { Diagnosed } \\
\text { by FNA and } \\
\text { biopsy }\end{array}$ \\
Menign findings & 15 & 21.4 & 100 \\
\hline Total & 70 & 15 & \\
\hline
\end{tabular}

BI-RADS, Breast Imaging Reporting and Data System; FNA, fine needle aspiration.

diagnosed sonographically as probably benign lesion (BI-RADS III) while five cases (7.14\%) with FABD were irregular hypoechoic masses on US and had spiculated outlines (highly suggestive of malignancy; BI-RADS V lesions), they were actually proved to be malignant on FNA and biopsy later on.

In 14 cases $(20 \%)$ with FABD, ultrasound showed heterogeneous ill-defined areas seen in locations corresponding to their FABD locations on mammogram. Pathological results showed fibrocystic changes in $8.5 \%$ (six cases), ductal carcinoma in situ (DCIS) in $2.85 \%$ (two cases), and invasive ductal carcinoma in $8.5 \%$ (six cases). In one case (1.4\%), the ultrasound showed thickened skin with edematous breast parenchymal tissue and inflammatory breast cancer was the histopathological result in this case. The summary was illustrated in Table 3.

An associated mass on mammogram was seen in six cases (two appeared benign and four proved to be malignant). In the malignant cases, the mass was irregular with ill-defined margin while in the benign cases, the mass was relatively well defined. Grouped microcalcification was observed in only two FABD and architectural distortion was observed in nine FABD areas. All these were proved to be malignant on subsequent pathological results. In this study, two cases with proved malignant FABD were palpable on clinical examination (Figs. 1-5).

\section{Discussion}

Many previous studies have reported that both malignant and benign breast lesions might be the cause of FABD on mammograms; indicating that it is challenging to decide which lesions need supplementary assessment with FNA or biopsy. ${ }^{15}$ There 


\begin{tabular}{|c|c|c|c|c|c|}
\hline \multicolumn{2}{|c|}{ Ultrasound findings } & US BI-RADS & No. & Percentage (\%) & $\begin{array}{l}\text { Pathological results } \\
\text { (biopsy) when indicated }\end{array}$ \\
\hline \multicolumn{2}{|c|}{ Normal fibroglandular tissue } & I & 25 & 35.7 & \\
\hline \multicolumn{2}{|c|}{ Ductal dilatation } & $\|$ & 12 & 17 & \\
\hline \multirow{3}{*}{ Cyst } & Simple cyst & $\|$ & 7 & 10 & \\
\hline & Complicated cyst & III & 1 & 1.4 & \\
\hline & $\begin{array}{l}\text { Complex cyst (with solid } \\
\text { component) }\end{array}$ & IV & 1 & 1.4 & Breast cancer \\
\hline \multirow{3}{*}{ Solid mass } & Probably benign mass & III & 4 & 5.7 & \\
\hline & Irregular hypoechoic mass & V & 5 & 7.14 & Invasive ductal carcinoma \\
\hline & & & 6 & 8.5 & Fibrocystic changes \\
\hline \multirow{2}{*}{\multicolumn{2}{|c|}{ Heterogeneous ill-defined area }} & IV & 2 & 2.8 & Ductal carcinoma in situ \\
\hline & & & 6 & 8.5 & Invasive ductal carcinoma \\
\hline \multicolumn{2}{|c|}{$\begin{array}{l}\text { Thick skin, edema, no mass, suspicious } \\
\text { axillary LN }\end{array}$} & IV-C & 1 & 1.4 & Inflammatory breast cancer \\
\hline \multicolumn{2}{|l|}{ Total } & & 70 & 100 & \\
\hline
\end{tabular}
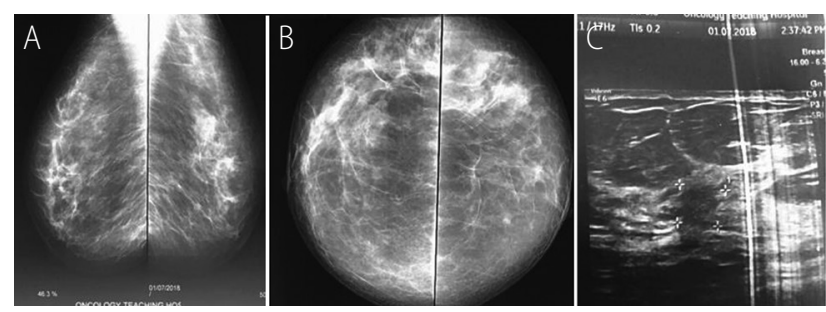

Fig. 1 A 52-year-old female presented with focal asymmetry at the upper outer quadrant of left breast at mediolateral and craniocaudal mammographic views (A and B). Subsequent ultrasound showed irregular hypoechoic lesion with ill-defined margin (C). FNA and biopsy showed invasive breast carcinoma.

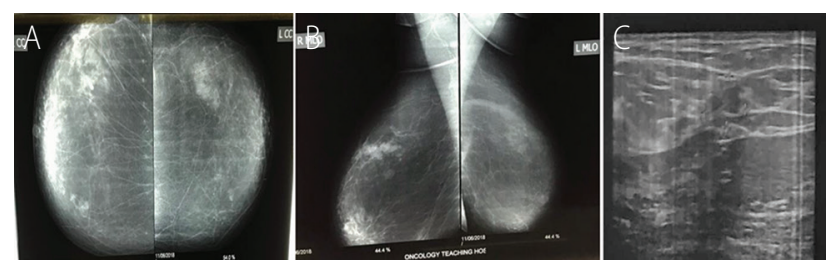

Fig. 2 A 56-year-old female referred for screening mammography. There is focal asymmetry at the upper outer quadrant of right breast in craniocaudal and mediolateral oblique views (A). Ultrasound showed heterogeneous ill-defined area (suspicious) (BI-RADS IV). Biopsy revealed fibrocystic changes.
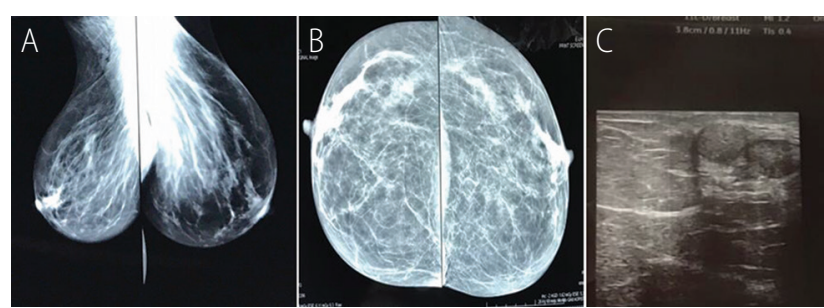

Fig. 3 A 60-year-old female presented with focal asymmetry at the retroareolar region of right breast in mediolateral oblique and craniocaudal views (A and B). Ultrasound revealed ductal dilatation with echogenic content inside (C). FNA result was consistent thick intraductal secretion.
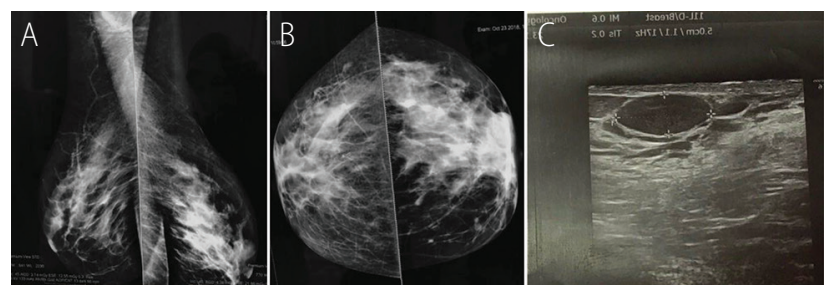

Fig. 4 A 43-year-old female presented for mammography. There is focal asymmetry at the retroareolar region of the left breast associated with relatively well circumscribed mass (A and B). Ultrasound $(C)$ showed well circumscribed oval shaped hypoechoic mass (typical benign ultrasound features) (BI-RADS III) and the patient was recommended to follow up.
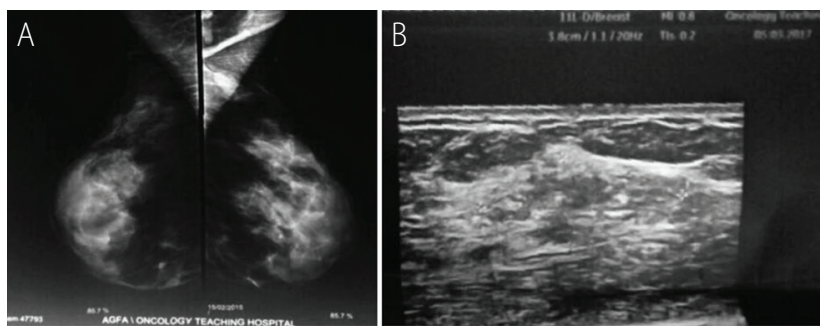

Fig. 5 A 44-year-old female presented with heterogeneously dense breast tissue on mammography. There is focal asymmetry at the upper outer quadrant of left breast on mediolateral oblique view (A) (craniocaudal view not available). Ultrasound at corresponding region showed heterogeneous ill-defined area (B). Biopsy revealed ductal carcinoma in situ (DCIS).

were many reported benign pathologies that cause FABD such as focal fibrotic changes of the breast, pseudoangiomatous hyperplasia and breast infections (tuberculous or pyogenic). ${ }^{18-20}$ These were consistent with the results of this study which revealed about $42.8 \%$ of FABD were actually caused by benign findings such as ductal dilatation, fibrocystic changes and probably benign lesions.

Some literatures reported that breast cancer was found in about $0-14 \%$ of asymmetric breast tissue. ${ }^{21}$ Others showed that invasive lobular carcinoma of breast was found in $15 \%$ of 
mammographic focal asymmetry, ${ }^{22}$ mucinous breast carcinoma in $4 \%$ of focal asymmetry, ${ }^{23}$ and tubular breast carcinoma in $5 \% .{ }^{24}$ Similarly, in this study, the results show that $21.4 \%$ of FABD were caused by malignant breast pathology and were: invasive ductal carcinoma in $15.6 \%$, DCIS in $2.8 \%$, inflammatory breast cancer in $1.4 \%$ and papillary breast cancer in $1.4 \%$ of cases.

About $35.7 \%$ of FABD cases included in this study had shown no abnormality on ultrasound. This was lower than those reported by Rissanen et al..$^{25}$ which showed that $53 \%$ of the FABD cases had normal ultrasound in their study which included 15 patients. Other researchers documented that all patients with FABD had normal ultrasounds; nevertheless the number of examined cases in their studies was too limited to detect abnormal ultrasonographic findings. ${ }^{19}$

All cases with FABD that were located in the upper inner quadrant (UIQ) in this study had revealed normal ultrasonographic findings except one case which showed heterogeneous ill-defined area and proved to be fibrocystic changes on FNA and biopsy. This was in agreement with the study of Zare and Langroudi ${ }^{26}$ which showed normal ultrasound in all FABD cases located in the UIQ.

In the current study, $80 \%$ of cases with retroareolar FABD showed ductal dilatation on ultrasound with significant relationship between retroareolar FABD and ductal dilatation $(P<0.05)$. These results were also consistent with the latter study which reported that $80 \%$ of retroareolar asymmetry was actually due to ductal dilatation. ${ }^{26}$

Architectural distortion was found in nine cases $(12.8 \%$ of total cases studied) and all were proved to be malignant on biopsy. Grouped microcalcifications were detected in two cases with FABD; both of which were also malignant as confirmed histopathologically by subsequent biopsy results. These findings were in accordance with the results recorded by Sperber et al. ${ }^{27}$

Four cases of FABD in this study were associated with an irregular mass on mammogram and another two cases of FABD were palpable on clinical examination. All these were histopathologically confirmed to be invasive ductal carcinoma by the corresponding excised biopsies; hence further evaluation of such cases would be mandatory. That was in agreement with the findings reported by Sperber et al. ${ }^{27}$ who believed that biopsy was absolutely necessary when focal asymmetry on mammography was palpable on clinical examination.
On the other hand, our results contradict those presented by Dennis et al. ${ }^{28}$ who proposed that a palpable abnormality with normal mammogram should not be biopsied. In such manner, many cases of breast cancer would be missed.

Many previous literatures had reported that focal asymmetry that was not present in the previous mammogram or became larger in size when compared with the previous mammogram (i.e. new or growing FABD) should have a special consideration as it would be more suspicious than focal asymmetry that was stable in size and appearance. ${ }^{13}$ In this study, all patients attended the breast unit for the first time (either for screening or diagnostic mammogram) and no previous mammograms were available for comparison.

If a focal asymmetry had its central part more dense than its periphery, then it should be regarded as suspicious because this concentrated density might be due to small hidden malignancy, ${ }^{29}$ whereas if the focal asymmetry had subtle lucencies within, it would be mostly a benign finding due to the superimposed fibroglandular tissue with the lucencies representing areas of interspersed fat.

\section{Conclusion}

The finding of FABD is common on mammography and is mostly a benign entity representing normal breast parenchymal tissue. FABD may indicate underlying hidden malignancy in the presence of superadded mammographic findings as ill-defined mass, architectural distortion or clustered microcalcification. Mammographic FABD that are not associated with the latter findings nor with a clinically palpable mass are most likely indicating a benign finding and could be followed safely.

\section{Acknowledgments}

The authors would like to acknowledge the support of the staff working in the Radiology and Pathology Departments of the Oncology Teaching Hospital and the Main Referral Center for Early Detection of Breast Tumors.

\section{Conflicts of interest}

The authors disclose that there are no conflicts of interest.

\footnotetext{
References

1. Bray F, Ferlay J, Soerjomataram I, Siegel RL, Torre LA, Jemal A. Global Cancer Statistics 2018: GLOBOCAN estimates of incidence and mortality worldwide for 36 cancers in 185 countries. CA Cancer J Clin. 2018:68:394-424.

2. Kelly KM, Dean J, Comulada WS, Lee SJ. Breast cancer detection using automated whole breast ultrasound and mammography in radiographically dense breasts. Eur Radiol. 2010;20:734-742.

3. Smith RA, Cokkindes V, Brooks D, Saslow D, Shah M, Brawley OW. Cancer screening in the United States, 2011: a review of current American Cancer Society guidelines and issues in cancer screening. CA Cancer J Clin. 2011;61:8-30.

4. Annual Report. Iraqi Cancer Registry 2015. Iraqi Cancer Board, Ministry of Health and Environment, Republic of Iraq, 2018.

5. Alwan NAS. Breast Cancer among Iraqi women: preliminary findings from a Regional Comparative Breast Cancer Research Project. J Glob Oncol. 2016;2:255-258.

6. Alwan NAS, Tawfeeq F, Maallah M, Sattar S. The stage of breast cancer at the time of diagnosis: correlation with the clinicopathological findings among Iraqi patients. J Neoplasm 2017;2:1-10.
}

7. Alwan NAS, Kerr D, Al-Okati D, Pezella F, Tawfeeg FN. Comparative study on the clinicopathological profiles of breast cancer among Iraqi and British patients. Open Public Health J. 2018;11:177-191.

8- Kopans DB, Smith RA, Duffy SW. Mammographic screening and "overdiagnosis". Radiology. 2011;260:616-620.

9. Schonberg MA, Ramanan RA, McCarthy EP, Marcantonio ER. Decision making and counseling around mammography screening for women aged 80 or older. J Gen Intern Med. 2006;21:979-985.

10. Fenton JJ, Egger J, Carney PA, Cutter G, D'Orsi C, Sickles EA, et al. Reality check: perceived versus actual performance of community mammographers. AJR Am J Roentgenol. 2006;187:42-46.

11. Samardar P, de Paredes ES, Grimes MM, Wilson JD. Focal asymmetric densities seen at mammography: US and pathologic correlation. Radiographics. 2002:22:19-33.

12. Kopans DB. Suspicious lesions and lesions with a high probability of malignancy. In: McAllister L, ed. Breast Imaging. $3^{\text {rd }}$ Ed.; Lippincott Williams \& Wilkins, Philadelphia, PA, 2007, pp. 513-553. 
13. Youk JH, Kim EK, Ko KH, Kim MJ. Asymmetric mammographic findings based on the fourth edition of BI-RADS: types, evaluation, and management. Radiographics. 2008;10:11.

14. Sickles EA, D'Orsi CJ, Bassett LW, et al. ACR BI-RADS: mammography. In: ACR BI-RADS Atlas, Breast Imaging Reporting and Data System. $4^{\text {th }}$ Ed.; American College of Radiology, Reston, Virginia, 2013.

15. Sickles EA. The spectrum of breast asymmetries: imaging features, work-up, management. Radiol Clin North Am. 2007:45:765-771, v.

16. Leung JW, Sickles EA. Developing asymmetry identified on mammography: correlation with imaging outcome and pathologic findings. AJR Am J Roentgenol. 2007;188:667-675.

17. Brenner RJ. Asymmetric densities of the breast: strategies for imaging evaluation. Semin Roentgenol. 2001;36:201-216.

18. Venta LA, Wiley EL, Gabriel H, Adler YT. Imaging features of focal breast fibrosis: mammographic-pathologic correlation of non-calcified breast lesions. AJR Am J Roentgenol. 1999;173:309-316.

19. Piccoli CW, Feig SA, Palzaao JP. Developing asymmetric breast tissue. Radiology. 1999;211:111-117.

20. Crowe DJ, Helvie MA, Wilson TE. Breast infection. Mammographic and sonographic findings with clinical correlation. Invest Radiol. 1995;30:582-587.

21. Dawson JS, Wilson AR. Short-term recall for 'probably benign' mammographic lesions detected in a three yearly screening programme. Clin Radiol. 1994:49:391-395.
22. Skaane P, Skjørten F. Ultrasonographic evaluation of invasive lobular carcinoma. Acta Radiol. 1999;40:369-375.

23. Goodman DN, Boutross-Tadross O, Jong RA. Mammographic features of pure mucinous carcinoma of the breast with pathological correlation. Can Assoc Radiol J. 1995:46:296-301.

24. Elson BC, Helvie MA, Frank TS, Wilson TE, Adler DD. Tubular carcinoma of the breast: mode of presentation, mammographic appearance and frequency of nodal metastases. AJR Am J Roentgenol. 1993;161:1173-1176.

25. Rissanen T, Pamilo M, Suramo I. Ultrasonography as a guidance method in the evaluation of mammographically detected nonpalpable breast lesions of suspected malignancy. Acta Radiol. 1998;39:292-297.

26. Zare Z, Faghihi Langroudi T. Findings of breast sonography in patients with focal asymmetric breast density on mammography. Iran Red Crescent Med J. 2011;13:404-406

27. Sperber F, Metser U, Gat A, Shalmon A, Yaal-Hahoshen N. Focal asymmetric breast density: mammographic, sonographic and pathological correlation in 97 lesions--a call to restrain biopsies. Isr Med Assoc J. 2007;9:720-723.

28. Dennis MA, Parker SH, Klaus AJ, Stavros AT, Kaske TI, Clark SB. Breast biopsy avoidance: the value of normal mammograms and normal sonograms in the setting of a palpable lump. Radiology. 2001;219:186-191.

29. Kopans DB. Interpreting the mammogram. In: McAllister L, ed. Breast Imaging.

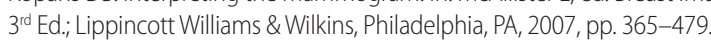

This work is licensed under a Creative Commons Attribution-NonCommercial 3.0 Unported License which allows users to read, copy, distribute and make derivative works for non-commercial purposes from the material, as long as the author of the original work is cited properly. 